PALEO

Revue d'archéologie préhistorique

26 | 2015

Varia

\title{
Olivier LE GALL (15 novembre 1954 à Langeac - 17 juin 2014 à Bordeaux)
}

Hélène Martin

\section{(2) OpenEdition}

1 Journals

Édition électronique

URL : http://journals.openedition.org/paleo/2874

DOI : $10.4000 /$ paleo.2874

ISSN : 2101-0420

Éditeur

SAMRA

\section{Édition imprimée}

Date de publication : 1 décembre 2015

Pagination : 11-15

ISSN : 1145-3370

\section{Référence électronique}

Hélène Martin, « Olivier LE GALL (15 novembre 1954 à Langeac - 17 juin 2014 à Bordeaux) », PALEO [En ligne], 26 | 2015, mis en ligne le 26 avril 2016, consulté le 08 juillet 2020. URL : http:// journals.openedition.org/paleo/2874 ; DOI : https://doi.org/10.4000/paleo.2874

Ce document a été généré automatiquement le 8 juillet 2020

\section{(c) (i) (9)}

PALEO est mis à disposition selon les termes de la licence Creative Commons Attribution - Pas d'Utilisation Commerciale - Pas de Modification 4.0 International. 


\section{Olivier LE GALL (15 novembre 1954 à Langeac - 17 juin 2014 à Bordeaux)}

\section{Hélène Martin}

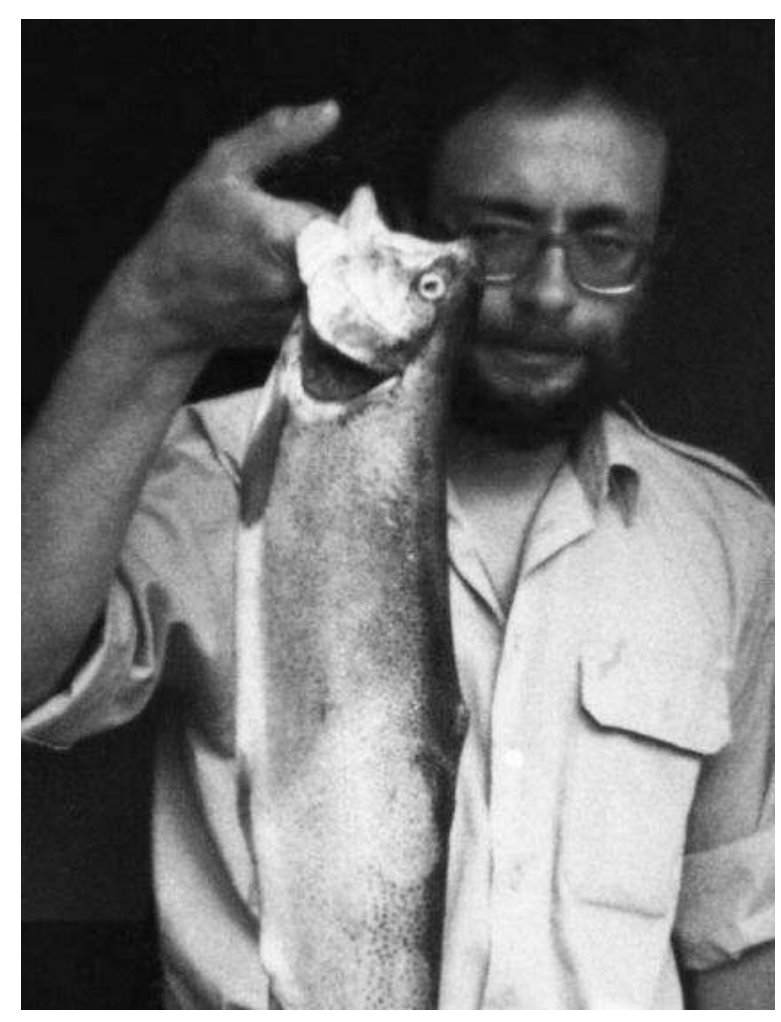

1 Le 17 juin 2014 s'en est allé Olivier le Gall. C'est pour moi, comme pour Pierre-Yves Demars qui l'a très bien exprimé dans un hommage publié dans Préhistoire du SudOuest, un ami qui disparaît. C'était également un mentor.

2 Il était devenu préhistorien, porté par son désir de comprendre : comprendre le monde où on vit, comprendre le monde d'où on vient et peut-être en tirer des leçons pour l'avenir. C'est ainsi qu'après des études de géologie, son penchant pour la Préhistoire et sa passion pour la pêche et les poissons l'avaient conduit à se spécialiser, sous la 
direction de F. Bordes et F. Prat, dans l'étude de l'ichtyofaune d'eau douce dans les sites préhistoriques. Ses travaux devaient se concrétiser par une thèse de Doctorat en Géologie du Quaternaire et Préhistoire soutenue à l'Université de Bordeaux I en 1982, puis par une thèse d'état en 1999 intitulée «Ichtyophagie et pêches préhistoriques. Quelques données de l'Europe occidentale». C'est en examinant les anneaux de croissance des vertèbres de poissons qu'il a pu définir leur saison de capture et appréhender ainsi les activités de prédation des pêcheurs préhistoriques. Cette approche initiatrice était fondée sur sa connaissance approfondie de l'éthologie animale et des milieux naturels, qu'il avait acquise au cours de sa jeunesse dans les gorges de l'Allier et dans les forêts de la Margeride. Passionné par tout ce qui touche aux relations Homme-animal et aux techniques de prédation, son besoin de comprendre s'exprimait également à travers la chasse qu'il pratiquait en étroite union avec ses chiens. Car Olivier n'était pas seulement un théoricien, il était aussi un praticien. De la pêche au saumon à la quête de la bécasse, il a aiguisé son regard de naturaliste et sa compréhension du comportement animal.

Ce sont ces dispositions particulières, cette rencontre entre le «flair» de l'homme de terrain et l'analyse rigoureuse du scientifique qui lui ont permis de se poser les bonnes questions relatives aux comportements de prédation des Paléolithiques. D'aborder des sujets aussi fondamentaux que ceux relatifs à l'invasion de l'Europe par les hommes anatomiquement modernes et les raisons pour lesquelles ils ont supplanté les Néanderthaliens. Et d'apporter des éléments de réponse: la présence du Chien, précieux auxiliaire, aux côtés des Aurignaciens, probablement excellents pratiquants de la cynégétique; leur arsenal technologique beaucoup plus vaste également. Autant d'éléments déterminants qui, s'ils ne sont bien évidemment pas les seuls, ont favorisé la suprématie des hommes anatomiquement modernes.

4 Ses idées originales, fruits d'une réflexion existentialiste approfondie et d'expériences acquises par une pratique du terrain qui manque tant à beaucoup de préhistoriens, son esprit de synthèse, n'avaient d'égal que son imagination. Or comme le dit si bien Albert Einstein "L'imagination est plus importante que le savoir, car le savoir est limité, alors que l'imagination embrasse l'univers entier ».

Olivier possédait les deux.

6 Alors bien sûr, il paraissait parfois un peu perdu dans un système où il est devenu plus important de faire une carrière plutôt que de la recherche. Où le principe de compétition entraîne inévitablement un formatage des cerveaux. Au formatage il répondait par la passion, au management et à la communication à outrance, par l'imagination et la rigueur.

7 Il est des personnes, rares, qui, par leur dimension scientifique et humaniste, se définissent comme des savants et dont la rencontre marque votre chemin, aussi bien professionnel que personnel. Olivier fut l'une d'elles. Il sut transmettre à ses étudiants ses valeurs profondes, cette curiosité et cette liberté de pensée indissociables de l'esprit de la recherche.

8 "N'essayez pas de devenir un homme qui a du succès, essayez de devenir un homme qui a de la valeur» disait encore Albert Einstein. Olivier restera pour moi, et pour d'autres assurément, cet homme-là. Il a été et restera un des grands préhistoriens de sa génération. 
9 Rédiger cet hommage, on s'en doute, est pour moi un moment chargé d'émotions. Je ne pensais pas avoir un jour à le faire. C'est aussi un moment riche de souvenirs.

Je revois ainsi Olivier, mettant ses pas dans ceux de nos ancêtres, suivre derrière ses chiens les mêmes gibiers de rêve. Je me souviens de lui au bord de la Truyère, du sifflement de sa soie et de l'arabesque qu'elle décrivait sur un fond de couchant, du posé de sa mouche sur l'eau souple des gobages.

11 Sa compréhension du monde passait par une profonde considération envers la nature, où chasseur et proie se rejoignent dans cette unité universelle qu'ont si bien su transcrire les peintres paléolithiques. Il savait que l'oubli de ce respect conduit inévitablement à ne plus respecter l'Homme. Et l'Homme a toujours été au cœur de ses interrogations.

12 Au revoir Olivier. Nos discussions fructueuses et ta capacité de synthèse me manquent. Le regard que tu portais sur le monde et plus spécialement sur le monde préhistorique aussi. Tu as laissé un grand vide. En nous tes amis bien sûr mais aussi pour la profession qui manque souvent d'un tel niveau d'excellence.

13 Nous nous retrouverons sans doute au détour d'une rivière. Accompagné de tes chiens, ton fantôme c'est sûr est parti à la pêche, rejoindre ces ombres que tu aimais tant.

\section{BIBLIOGRAPHIE}

LE GALL 0. 1981 - L’ichtyofaune dulcicole dans les gisements préhistoriques. Quaternaria, vol. 23, p. 219-232.

LE GALL Olivier - 1982 - Les Poissons de quelques gisements préhistoriques du Sud-Ouest de la France : étude ostéologique. Intérêt paléoécologique et palethnologique. Thèse présentée à l'Université de Bordeaux I pour l'obtention du titre de Docteur en géologie du quaternaire et préhistoire. 2 vol. (184 p-104 pl.).

LE GALL 0.1983 - Présence de vestiges de Saumon atlantique dans un site épipaléolithique et mésolithique du bassin versant méditerranéen. Bulletin de la Société anthropologique du Sud-Ouest, tome XVIII, $\mathrm{n}^{\circ} 1$, p. 52-55.

LE GALL 0. 1984 - L'Ichtyofaune d'eau douce dans le sites préhistoriques : ostéologie, paléoécologie, palethnologie. Paris : CNRS. 1 vol. (196 p.). (Cahiers du quaternaire ; 8).

LE GALL O. 1984 - L'Exploitation de l'ichthyofaune par les Paléolithiques : quelques exemples. In : DESSE-BERSET Nathalie (dir.). 2èmes Rencontres d'archéo-ichthyologie : table ronde Sophia-AntipolisValbonne, 14-16 oct. 1983. Paris : CNRS. (Notes et monographies techniques ; 16). p. 89-112.

LE GALL O. (Dir. Fouille) 1984 - Cerzat : Le Pie du Roi. Gallia Préhistoire, Tome 27, fasc. 2, p. 324.

LE GALL 0. 1986 - Les os de poisson contenus dans la vase, Bulletin de la Société de Borda, 1er trimestre, p. 71-76. 
LE GALL O. 1989 - Les poissons de la grotte Vaufrey. In : RIGAUD J. Ph. (dir.). La Grotte Vauffrey à Cénac et Saint-Julien (Dordogne). Paris : Société préhistorique française. (Mémoires ; XIX). p. 351356.

LE GALL O. 1989 - Analyse palethnologique de l'ichtyofaune. In: RIGAUD J. P. (dir.). La Grotte Vauffrey à Cénac et Saint-Julien (Dordogne)és humaines. Paris : Société préhistorique française. (Mémoires ; XIX). p. 565-568.

LE GALL 0. 1991 - Pêcheurs mésolithiques et néolithiques : similitudes ou différences de comportement. In: Mésolithique et néolithisation en France et dans les régions limitrophes : actes du $113 e$ Congrès national des Sociétés savantes, Strasbourg, 5-9 avril 1988. Paris : Comité des travaux historiques et scientifiques. p. 99-111.

LE GALL O. 1992 - Poissons et pêches au Paléolithique : quelques données de l'Europe occidentale, L'Anthropologie, vol. 96, n 1, p. 121-134.

LE GALL O. 1992 - Les Magdaléniens et l'ichtyofaune dulçaquicole. In : Le Peuplement magdalénien : paléogéographie physique et humaine : Colloque de Chancelade, 10-15 octobre 1988. Paris : Comité des travaux historiques et scientifiques. p. 277-285.

LE GALL O. 1992 - Les poissons des niveaux gravettiens de Temnata. In: Temnata Cave: excavations in Karlukovo karst area, Bulgaria. vol. 1-Part 1. Cracovie : Jagellonian University Press. p. 137-140.

LE GALL O. 1993 - La pêche et les poissons pendant le Paléolithique en Périgord. In : DELLUC B. et G. Le Périgord préhistorique. Montignac : Reflets du Périgord. p. 61-65.

LE GALL O. 1993 - Evolution des pêches de l'Epipaléolithique au Néolithique ancien, Préhistoire, anthropologie méditerranéennes, $\mathrm{n}^{\circ}$ 2, p. 135-142.

LE GALL 0. 1994 - Quelques remarques sur l'adaptation à court et à long termes chez les poissons d'eau douce du sud de la France. In: VAN NEER W. (dir.). Fish exploitation in the past: proceedings of the 7th meeting of the ICAZ, Fish Remains Working Group. Tervuren : Musée royal de l'Afrique centrale. p. 91-98.

LE GALL O. 1995 - Ichthyologie. In: STRAUS L.G. (dir.). Les derniers chasseurs de rennes du monde pyrénéen. L'abri Dufaure : un gisement tardiglaciaire en Gascogne. Paris : Société préhistorique française. (Mémoires ; XXII). p. 247-248.

LE GALL O. 1995 - Etude des poissons. In : SÉRONIE-VIVIEN M.R. (dir.). La Grotte de Pégourié, Caniacdu-Causse (Lot). Cabrerets : Association des amis du Musée Amédée Lemozi. (Préhistoire quercinoise ; Supplément 2). p. 149-154.

LE GALL 0. 1995 - Els peixos de la Balma de la Margineda (neolític i mesolític) = Les poissons de la Balma de la Margineda (Néolithique et Mésolithique). In : GUILAINE J. (dir.). Les Excavacions a la Balma de la Margineda (1979-1991). vol. III. Andorra : Institut d'Estudis Andorrans. p. 98-110.

LE GALL O. 1997 - A propos des harpons magdaléniens. Bulletin de la Société d'anthropologie du SudOuest, Tome XXXII, 3e et 4e trimestres, p. 111-120.

LE GALL O. 1998 - Aperçu des pêches maritimes préhistoriques en Europe occidentale : Méditerranée nord-occidentale, Atlantique nord, Manche, Mer du Nord, Baltique. In : CAMPS Gabriel (dir.). L'Homme préhistorique et la mer : 120e Congrès national des sociétés historiques et scientifiques, Aix-en-Provence, 23-26 octobre 1995. Paris : Comité des travaux historiques et scientifiques. p. 377-386.

LE GALL 0. 1999 - Éléments de réflexion sur la pêche dans le bassin méditerranéen nordoccidental pendant le développement des faciès leptolithiques. In : SACCHi Dominique (dir.). Les Faciès leptolithiques du Nord-Ouest méditerranéen : milieux naturels et culturels : XXIVe Congrès 
préhistorique de France, Carcassonne, 26-30 sept. 1994. Paris : Société préhistorique de France. p. 251-265.

LE GALL O. 1999 - Ichtyophagie et pêches préhistoriques : quelques données de l'Europe occidentale. Thèse présentée à l'Université de Bordeaux 1 pour obtenir le grade de Docteur d'État es sciences. 1 vol. (473 p.).

LE GALL 0. 1999 - Les poissons. In : CHAUCHAT Claude (dir.). L'habitat magdalénien de la Grotte du Bourrouilla à Arancou (Pyrénées-Atlantiques). Paris : CNRS. (Gallia préhistoire ; 41). p. 129-132.

LE GALL 0. 2000 - L'origine et l'histoire des aloses. In : BAGLINIÈRE J. L. (dir.), ELIE P. (dir.). Les aloses (Alosa alosa et Alosa fallax spp. ) : écobiologie et variabilité des populations. Paris : Institut national de la recherche agronomique. (Hydrobiologie et aquaculture). p. 127-136.

LE GALL 0. 2001 - Les Représentations de poissons dans l'art mobilier magdalénien : une expression de l'importance culturelle de la pêche. Préhistoire du Sud-Ouest, n 8, fasc. 1, p. 55-69.

LE GALL 0. 2003 - Des Magdaléniens et... des poissons. In : COSTAMAGNO Sandrine (dir.), LAROULANDIE V. (dir.). Mode de vie au Magdalénien : apports de l'archéozoologie : actes du XIVème Congrès UISPP, Université de Liège, Belgique, 2-8 septembre 2001. Section 6, Paléolithique supérieur. Oxford : Archaeopress. (BAR International series ; 1144). p. 119-128.

LE GALL 0. 2003 - La Squelettochronologie appliquée aux poissons : une méthode de reconnaissance des saisons de capture. Préhistoire du Sud-Ouest, n 10, p. 9-24.

LE GALL 0. 2005 - Un contrefort du Massif central du Gravettien à l'Azilien : indices d'occupations humaines et de migrations animales fondés sur les « saisonnalités » In : JAUBERT J. (dir.), BARBAZA M. (dir.). Territoires, déplacements, mobilité, échanges durant la Préhistoire : terres et hommes du Sud : actes du 126e Congrès national des Sociétés historiques et scientifiques. Paris : Comité des travaux historiques et scientifiques. p. 265-278.

LE GALL 0. 2005 - Le galet gravé de la Honteyre (Tuzan, Gironde). Préhistoire du Sud-Ouest, $\mathrm{n}^{\circ} 12$ (2), p. 175-188.

LE GALL 0. 2006 - Les apports de la squelettochronologie en archéologie préhistorique : quelques exemples. In: BRESSY C. (dir.), BURKE A. (dir.), CHALARD P. (dir.), MARTIN H. (dir.). Notions de territoire et de mobilité : exemples de l'Europe et des premières nations en Amérique du Nord avant le contact européen : actes des sessions présentées au Xe congrès annuel de l'Association européenne des Archéologues (EAA), Lyon, 8-11 septembre 2004. Liège : Université. (ERAUL ; 116). p. 51-62.

LE GALL 0. 2008 - Els peixos de la Balma de la Margineda (Capes de la 7 a la 10). In GUILAINE J. (dir.), BARBAZA M. (dir.), MARTZLUFF M. (dir.). Les excavacions a la Balma de la Margineda (1979-1991), vol. IV. Andorra : Ministeri del Portaveu, Cultura i Ensenyament Superior. p. 532-539.

LE GALL O. 2008 - Les Poissons des eaux douces pléistocènes sont-ils des indicateurs paléoclimatiques ? : une approche élargie à l'Europe. In : BEAREZ Philippe (dir.), GROUARD Sandrine (dir.), CLAVEL Benoît (dir.). Archéologie du poisson : 30 ans d'archéo-ichtyologie au CNRS ; hommage aux travaux de Jean Desse et Nathalie Desse-Berset; actes des XXVIIIe Rencontres Internationales d'archéologie et d'histoire d'Antibes, 18-20 octobre 2007. Antibes : APDCA. p. 311-326.

LE GALL 0. 2008 - Ichtyofaunes et pêches à la grotte Walou. In : DEWEZ M. (dir.) ; CREVECOEUR I., FRANCIS A., KLARIC L. [et al]. Recherches à la grotte Walou à Trooz (Belgique) : second rapport de fouille. Oxford : Archaeopress. (BAR International series ; 1789). p. 57-68.

LE GALL O. 2009 - Les poissons des Peyrugues (Lot). Préhistoire du Sud-Ouest, vol. 17 (2), p. 205-216.

LE GALL 0. 2009 - Archéo-ichtyologie et pêches préhistoriques : résultats et perspectives. Archéopages, vol. 26, p. 52-55. 
LE GALL 0. 2010 - Influences des glaciaires-interglaciaires sur les ichtyofaunes des eaux douces européennes. Quaternaire, vol. 21, nº 3, p. 203-214.

LE GALL O. 2010 - Les poissons de l'aven de Romain-la-Roche (Doubs, France). Revue de Paléobiologie, décembre 2010, $\mathrm{n}^{\circ} 29$ (2), :p. 859-860.

LE GALL 0. 2011 - Origines des vestiges de poissons d'eaux douces dans les sites paléolithiques : un point sur la question. In : LAROULANDIE V., MALLYE J.-B., DENYS C. Taphonomie des petits vertébrés : référentiels et transferts aux fossiles : actes de la table ronde du RTP Taphonomie, Talence 20-21 octobre 2009. Oxford : Archaeopress. (BAR International Series ; 2269). p. 153-166.

LE GALL O., MARTIN H. 1996 - Pêches et chasses aux limites Landes/Pyrénées : quelques éléments de réflexion fondés sur les saisonnalités. In : DELPORTE H. (dir.), CLOTTES J. (dir.). Pyrénées préhistoriques : arts et sociétés : actes du 118e Congrès national des Sociétés historiques et scientifiques, Pau, 25-29 oct. 1993. Paris : Comité des travaux historiques et scientifiques. p. 163-172.

LE GALL O., PANNOUX Ph. 1994 - Les poissons de Pont d'Ambon. Gallia préhistoire, n 36, p. 113-126.

LE GALL O., RAYNAL J.-P. 1986 - Cerzat (Haute-Loire) : « Le Pié du Roy ». Un site préhistorique dans un volcan. Revue Archeologique du Centre de la France, Tome 25, volume 1, p. 99-100.

LE GALL O., RAYNAL J.-P. 2005 - Préhistoires de pêche : exposition présentée par le Centre de Documentation et de Recherches Archéologiques Départemental (CDERAD) [en ligne]. CCSD/ Archéo-Logis (HAL), 2005. [document consulté le 22/09/2015].Disponible à l'adresse : https:// halshs.archives-ouvertes.fr/halshs-00004299/document

LE GALL O., ALTUNA J., STRAUS L.G. 1994 - Les Faunes mésolithiques et néolithiques de Vidigal (Alentejo, Portugal), Archaeozoologia, n 1, p. 59-72.

LE GALL O., MARTIN H., DEMARS P.-Y. 2013 - Le passage de Néandertal à l'Homme anatomiquement moderne : une révolution technologique ? In : JAUBERT J., FOURMENT N., DEPAEPE P. Transitions, ruptures et continuité en Préhistoire : actes du XXVIIe Congrès préhistorique de France, Bordeaux-Les Eyzies, 31 mai-5 juin 2010, Volume 1. Paris : Société préhistorique française. p. 47-58.

LE GALL O., DEMARS P.-Y., JARRY M., MARTIN H. 2013 - Saisons et proies : réflexions sur les notions de prédation(s) et de territoire(s). In: JARRY M. (dir.), BRUGAL, J.-Ph. (dir.), FERRIER, C. (dir.). Modalité d'occupation et exploitation des milieux au Paléolithique dans le Sud-Ouest de la France : l'exemple du Quercy. Actes de la session C67, XVème Congrès mondial de l'UISPP, Lisbonne, septembre 2006. Les Eyzies-de-Tayac : Musée national de Préhistoire. (PALEO ; suppl. 4).p. 419-437.

LE GALL O., STRAUS L. G., VIERRA B. J., ALTUNA J. - 1992 - Ichthyofaunas and seasonality at Vidigal (Alentejo, Portugal). Mesolithic Miscellany, vol. 13, $\mathrm{n}^{\circ}$ 2, p. 13-18.

\section{En collaboration}

BAFFIER D., BUISSON D., DELPORTE H., FRITZ C., GAILLI R., GUY E., LE GALL O., PAILHAUGUE N., ROBERT R. SAUVET G., TOSELLO G., WELTE A.-C. 2003 - Tubes et os d'oiseaux In : CLOTTES J. (dir.), DELPORTE Henri (dir.). La grotte de La Vache (Ariège) : Fouilles Romain Robert. II-L'art mobilier. Paris : RMN. p. 323-383.

BOEUF O., BONIFAY E., BONIFAY M.-F., BOURDELLE Y., CARRE F., CONSIGNY A., DAUGAS J.-P., DELPORTE H.i, LE GALL O., LIABEUF R., RAYNAL J.-P. 1988 - Les peuplements paléolithiques du 
Massif central : séance décentralisée, Le Puy-en-Velay, 8 et 9 octobre 1988 : livret-guide des excursions. Paris : Société préhistorique française. 1 vol. (40p.).

DACHARY M., MERLET J.-C., MIQUÉOU M., MALLYE J.-B., LE GALL O., EASTHAM A. 2013 - Les occupations mésolithiques de Bourrouilla à Arancou (Pyrénées-Atlantiques, France) : une séquence clef de la fin du Magdalénien pyrénéo-cantabrique. PALEO, n² 24, p. 79-102.

DACHARY M. (dir.), CHAUVIÈRE F.-X., COSTAMAGNO S., DAULNY L., EASTHAM A., FERRIER C., FRITZ C., LE GALL O. 2007 - Arancou : Bourrouilla. In : Bilan scientifique de la région Aquitaine 2005. Bordeaux : Service Régional de l'Archéologie Aquitaine. p. 181-183.

DELPECH F., LE GALL O. 1983 - La faune magdalénienne de la grotte des Eglises (Ussat, Ariège). Préhistoire ariégeoise, Tome XXXVIII, p. 91-118.

DELPECH F., LE GALL O. 1986 - International Council for archaeozoology : Bordeaux-Talence, 1986 : livret-guide des excursions du 28 août. Talence : Institut de Quaternaire. 1 vol. (152 p.)

DELPECH F., DONARD E., GILBERT A., GUADELLI J.-L., LE GALL O., MARTINI-JACQUIN A., PAQUEREAU M.-M., PRAT F., TOURNEPICHE J.-F. 1983 - Contribution à la lecture des paléoclimats quaternaires d'après les données de la paléontologie en milieu continental. In : Paléoclimats : journées de Bordeaux, 30-31 mai 1983. Paris : CNRS. (Cahiers du Quaternaire ; HS). p. 165-177.

DEMARS P.-Y., LE GALL O., MARTIN H. 2007 - Saisonnalité, mobilité et spécialisation des sites : une approche polythématique. In : BEAUNE Sophie A. de (dir.). Chasseurs-cueilleurs : comment vivaient les hommes du Paléolithique supérieur - méthodes d'analyse et d'interprétation en préhistoire. Paris : CNRS. p. 99-115.

DUCASSE S. (dir.), RENARD C. (dir.) ; ASTRUC G., AVERBOUH A., BRUXELLES L., CASTEL J.-Ch., CHALARD P., CLOTTES J., DESCLAUX E., FOURMENT N., FRITZ C., GIRAUD J.-P., HENRI-GAMBIER D., KERVAZO B., KONIK S., LE GALL O., LE GUILLOU Y., LELOUVIER L.-A., MARTIN B., MARTIN H., MORALA A., OBERLIN Ch., PéTILLON J.-M., POMIES M.-P., SERVELLE Ch., TABORIN Y., TURQ A., VALLADAS H., VIGNAUD C., VILLOTTE S. 2012 - De 20000 à 18000 BP en Quercy : apports de la séquence du Cuzoul de Vers à la compréhension de l'évolution des comportements socioéconomiques entre Solutréen récent et Badegoulien. In : CLOTTES Jean (dir.), GIRAUD Jean-Pierre (dir.), CHALARD Pierre (dir.). Solutréen et Badegoulien au Cuzoul de Vers : des chasseurs de Rennes en Quercy. Liège: université. (ERAUL; 131). p. 459-471.

GEDDES D., GUILAINE J., COULAROU J., LE GALL O., MARTZLUFF M. 1989 - Postglacial environments, settlement and subsistence in the Pyrenees: the Balma Margineda, Andorra. In: Bonsall, Clive (dir.). The Mesolithic in Europe (UISPP, 1985); Edinburgh: John Donald Publishers Ltd. p. 561-571.

GUADELLI J.-L., LE GALL O., MARTIN H. 1983 - L'Homme et l'animal. In : Les Inédits de la préhistoire auvergnate, Catalogue de l'exposition, Musée Bargoin, Clermont-Ferrand, 12 juil.-15 oct. 1983. [s.l.] : [s. n.]. p. 41-47.

JARRY M., MARTIN H., DEMARS P.-Y., LE GALL O. - 2008 - Territoires et saisons au Paléolithique : éléments de réflexion. Archéopages, ${ }^{\circ}$ 21, p. 6-15.

JAUBERT J., BRUGAL J.-Ph., VENDITTI F., HERNANDEZ M., BOUDADI-MALIGNE M., ARGANT A., BAHAIN J.-J., COUCHOUD I., FALGUÈRES Ch., FERNANDEZ Ph., JEANNET M., KERVAZO B., LATEUR N., LE GALL O., LÉCUYER Ch., LEMORINI C., MARTIN H., MERCIER N., MOURRE V. 2012 Coudoulous I in Quercy (SW France) : from marginal scavenging to a kill-butchery site for Preneandertal. In: European Society for the study of Human Evolution (ESHE): 2nd Annual Meeting, Bordeaux, France, 21 and 22 September, 2012. Leipzig : ESHE. p. 109 
MARTIN H., LE GALL O. 1989 - La faune mésolithique du gisement de la Doue (Corrèze). Implications palethnologiques. Archaeozoologia, vol. III (1.2), p. 263-274.

LANGLAIS M. (dir.), LAROULANDIE V. (dir.) ; BRUXELLES L., CHALARD P., COCHARD D., COSTAMAGNO S., DELFOUR G., KUNTZ D., LE GALL O., PETILLON J.-M., QUEFFELEC A. 2009 - Les fouilles de la grotte-abri de Peyrazet (Creysse, Lot) : nouvelles données pour le Tardiglaciaire quercinois. Bulletin de la Société préhistorique française, vol. 106, n 1, p. 150-152.

MARTIN H., LE GALL O. 1987 - Le comportement des chasseurs mésolithiques de La Doue (Corrèze). Les indices fournis par la faune. Revue archéologique du centre de la France, Tome 26, volume 1, p. 67-74.

MARTIN H., LE GALL O. 2000 - La Faune : un indicateur des comportements humains. La chasse du cerf au Post-Paléolithique : deux exemples. In BELLIER C., CATTELAIN P., OTTE M. - La Chasse dans la Préhistoire : actes du colloque international de Treignes, 3-7 octobre 1990. Liège : Université. (ERAUL ; 51). p. 364-369.

RAYNAL J.-P., LAFARGE A., RÉMY D., DELVIGNE V., GUADELLI J.-.Luc, COSTAMAGNO S., LE GALL O., DAUJEARD C., VIVENT D., FERNANDES P., LE CORRE-LE BEUX M., VERNET G., BAZILE F., LEFÈVRE D. 2014 - Datations SMA et nouveaux regards sur l'archéo-séquence du Rond-du-Barry (Polignac, Haute-Loire). Compte rendu Palevol, vol. 13, n 7, p. 623-636.

SACCHI D. (dir.) ; CREGUT-BONNOURE E., HEINZ Ch., LE GALL O., MAURE M., VERNET J.-L., VILETTE Ph. 1992 - Un site paléolithique supérieur de moyenne altitude dans les Pyrénées : la Cauna de Belvis (France). Preistoria Alpina, vol. 28, n² 2, p. 59-90. 